\title{
Klebsiella Pneumoniae Liver Abscess Syndrome - A Challenge for Contrast-Enhanced Ultrasound
}

\section{Introduction}

Invasive liver abscess syndrome (ILAS) is caused by strains of hypervirulent Klebsiella pneumoniae (hvKp) and has emerged as the leading cause of liver abscesses in immuno-competent patients (L.K. Siu et al. Lancet Infect Dis 2012; 12: 881-87). ILAS is frequently associated with metastatic spread including the eyes, lungs, and the central nervous system. The morbidity and mortality of affected patients are increased compared to liver abscesses of other origin, especially in cases with concomitant diabetes mellitus (J. E. Choby et al. J Intern Med 2020; 287(3): 283-300). Immediate diagnosis and early intervention are essential for an optimal outcome. Therefore, ultrasound plays a crucial role if hvKp is suspected. However, experience with the characteristics of such abscesses on contrast-enhanced ultrasound (CEUS) is very limited.

\section{Case Description}

A 69-year-old woman presented after a collapse to the emergency department. She complained of back pain, but also reported fever and chills. She had received a longterm steroid therapy for rheumatoid arthritis and had immigrated from Vietnam 10 years ago. The patient remembered a conservatively treated liver abscess a long time ago.

Laboratory results showed an elevated leucocyte count, C-reactive protein, and aspartate aminotransferase. Magnetic resonance (MR) imaging of the spine showed a stable lumbar fracture, but also revealed a lesion of the right liver lobe, which could not be further characterized by the applied MR protocol. The patient was hospitalized for conservative treatment of the spinal fracture. After four days, gastroenterologists were consulted due to worsening of the patient's general condition.

The ultrasound examination detected a large hypoechoic inhomogeneous lesion in the lateral segments of the right liver lobe with irregular demarcation and solid morphology ( $\triangleright$ Fig. 1a). Duplex ultrasonography showed significant perfusion of the lesion, which raised the suspicion of malig-

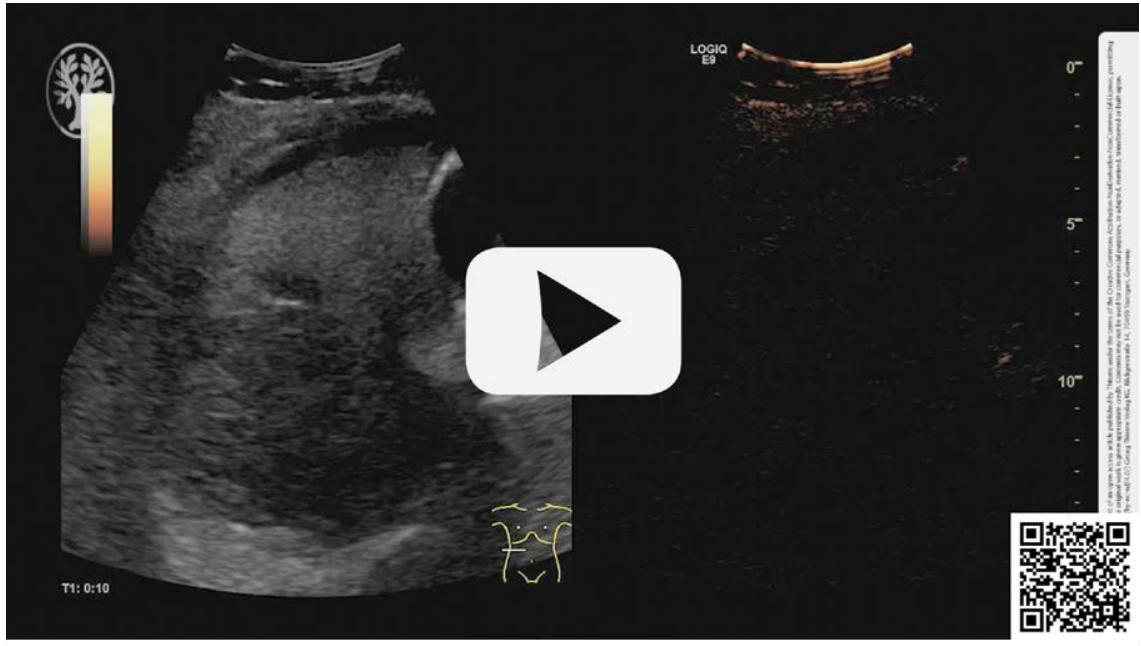

- Suppl Video 1

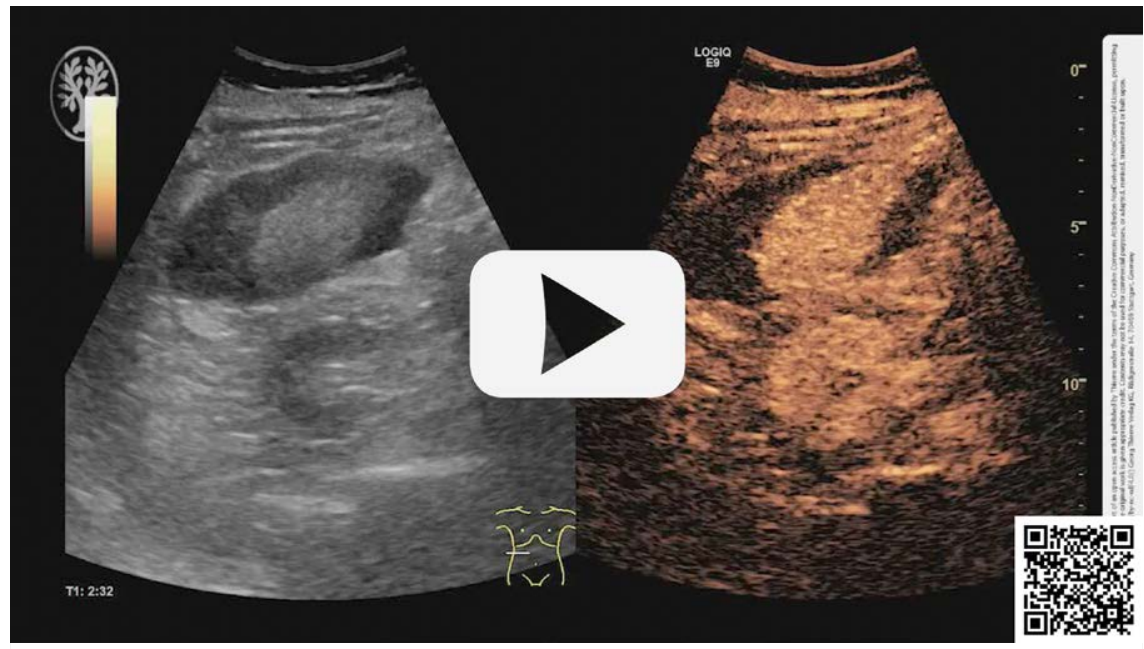

- Suppl Video 2
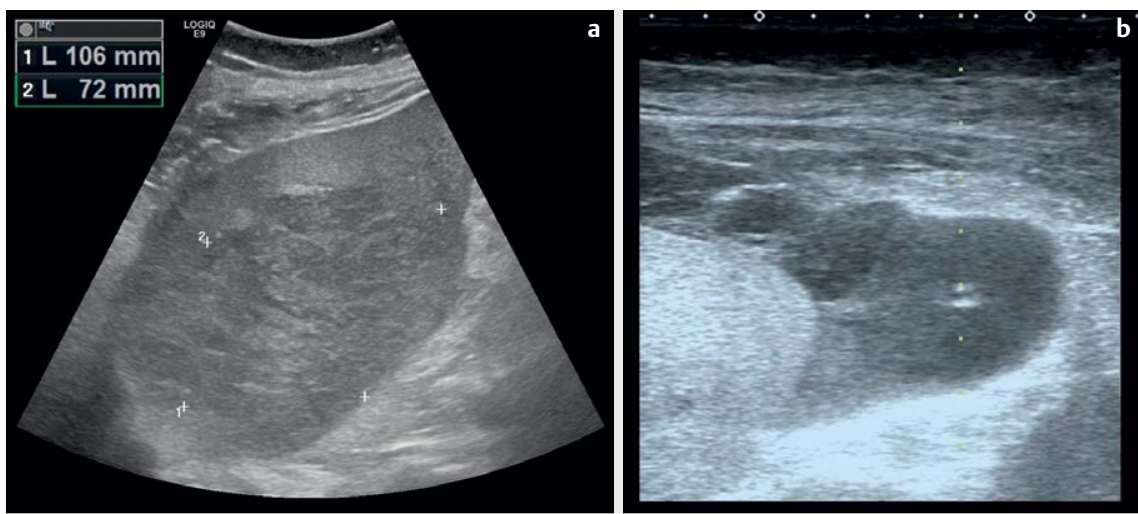

- Fig. 1 B-mode ultrasound of liver lesion. The size of the lesion in transverse section is marked a. Longitudinal B-mode ultrasound of lower liver margin showing ultrasound-guided puncture of free perihepatic fluid? $\mathbf{b}$. 

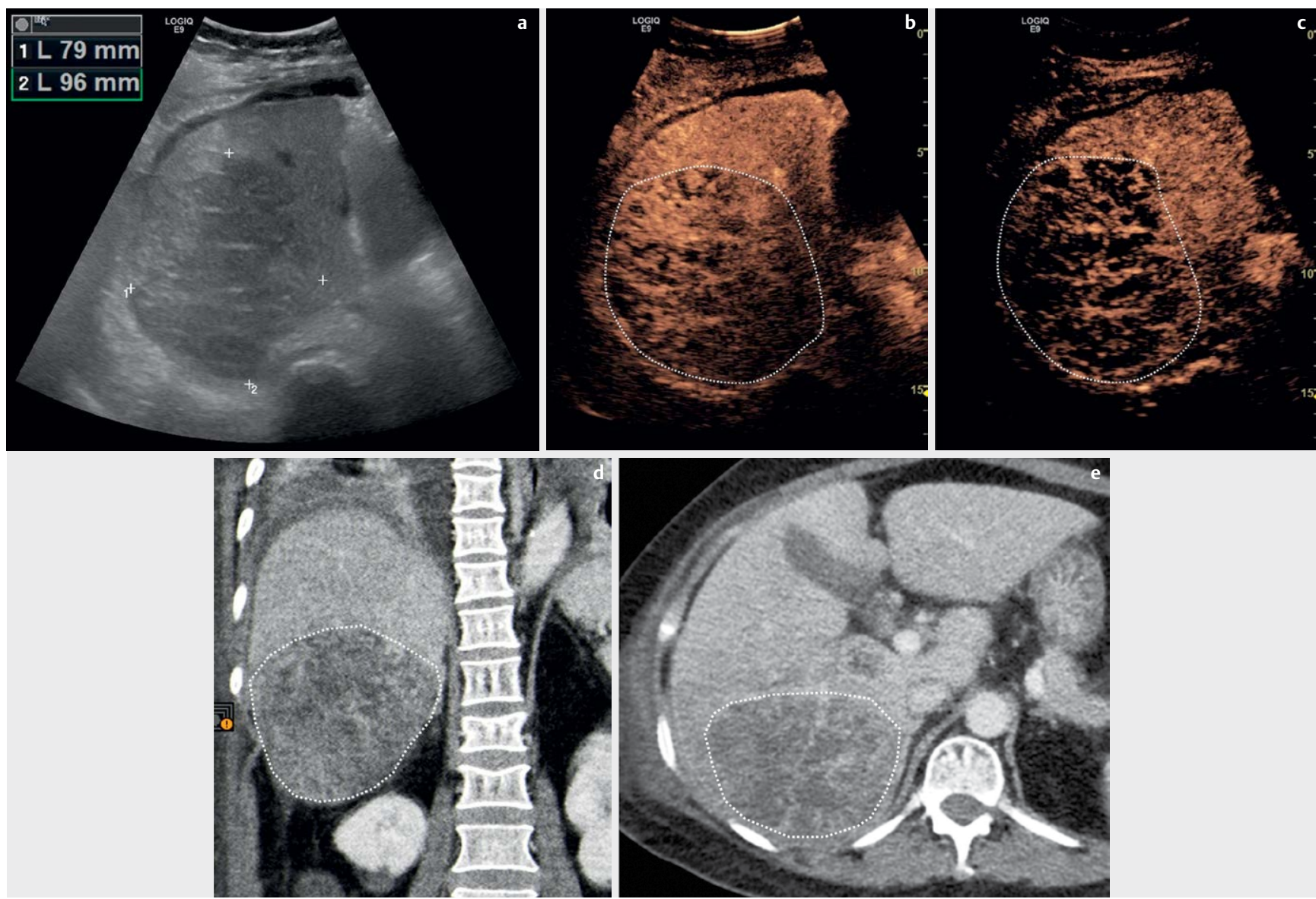

- Fig. 2 Transverse B-mode ultrasound of the right liver lobe $\mathbf{a}$ and contrast-enhanced ultrasound in the early $\mathbf{b}$ and late portal venous phases c after application of contrast agent (SonoVue ${ }^{\circledR}$ ). Incomplete arterial enhancement followed by a rapid but incomplete wash-out with residual septa was observed. Coronal $\mathbf{d}$ and transverse $\mathbf{e}$ planes on contrast-enhanced computed tomography imaging of the lesion in the portal venous phase and after 3D reconstruction.

nancy. Hence, CEUS was performed and revealed incomplete arterial enhancement followed by a rapid but incomplete washout with residual septa ( $\triangleright$ Figs. 2a-c, arterial phase: supplementary video $\mathbf{1}$; late phase: supplementary video 2 ). This pattern was compatible with a malignant liver tumor, although the clinical picture indicated an infectious complication.

Because the patient's condition deteriorated rapidly with shortness of breath, hypotension, and somnolence, computed tomography $(C T)$ was performed and confirmed the inhomogeneous and hypoenhancing lesion of the right liver lobe but did not show any evidence of metastatic disease ( $\triangleright$ Figs. $\mathbf{2 d}$ and e). In concordance with the CEUS findings, the irregular demarcation of the tumor and the vascularization pattern indicated a malignant liver tumor, i. e., intrahepatic cholangiocarcinoma or poorly differ- entiated hepatocellular carcinoma. A potential liver abscess was discussed in the CT report, but deemed unlikely. In this situation, the further treatment decision depended on immediate differential diagnosis between a benign infectious lesion and an advanced malignant tumor with superinfected necrosis.

Therefore, duplicate use bedside ultrasound-guided puncture of the narrow perihepatic ascites collection was performed ( $\vee$ Fig. 1b) and detected a high leukocyte count and gram-negative strains in the abdominal fluid. Given the life-threatening condition, we decided against additional percutaneous biopsy or further MRI imaging and established the clinical diagnosis of a liver abscess. Antibiotic treatment with piperacillin/tazobactam was initiated. Due to the solid appearance of the lesion, drainage therapy was not feasible. Thus, urgent surgical intervention revealed a purulent peritoneum and alteration of the liver parenchyma due to inflammation. A resection of the affected liver segments was performed. Histologic evaluation of the hepatic specimen confirmed a pyogenic liver abscess with an infiltrative appearance and without a sharp demarcation from the hepatic tissue ( $\triangleright$ Figs. 3a and $\mathbf{b}$ ). This was consistent with the final microbiological result showing hypermucoviscous Klebsiella pneumonia.

We adjusted the antibiotic treatment to cefotaxime and ciprofloxacin. During the further course, the patient suffered from prolonged wound healing but ultimately recovered well. An ultrasound control examination four months later did not show any remaining abscess formations. 


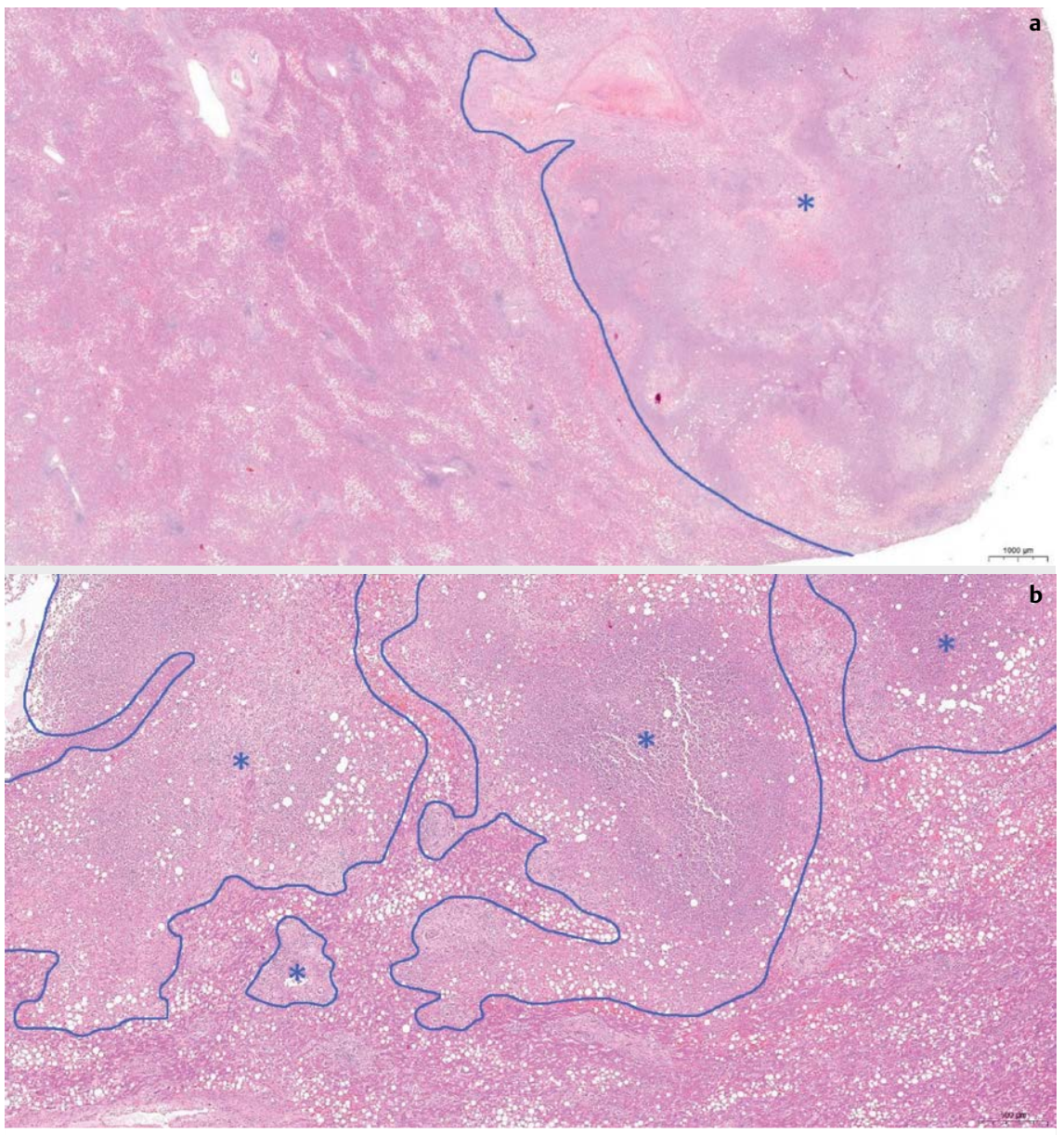

- Fig. 3 Histologic view of hepatic specimen. Overview with demarcation of liver abscess $\left({ }^{*}\right)$ a. Magnification of a different field of view with irregular spread of abscess formations $\left({ }^{*}\right)$, surrounded by hepatic tissue with altered architecture $\mathbf{b}$. of the abscess formation inside the liver parenchyma. We assume that impaired portal venous perfusion and shunts could be responsible for the rapid washout phenomenon in both CEUS and CT scans. Such an imaging pattern represents a diagnostic challenge in life-threatening scenarios with the need for an urgent decision. In the present case, a malignant tumor would have resulted in palliative treatment with best supportive care.

In conclusion, the serious prognosis of hvKp ILAS demands an urgent diagnostic approach using ultrasound. However, the CEUS pattern may suggest malignancy and critical interpretation in the clinical context is required.

\section{Summary}

- Klebsiella pneumoniae-associated ILAS represents an emerging disease and should be considered in patients with liver tumors, especially in cases with Asian origin or history of liver abscesses.

- Contrast-enhanced ultrasound (CEUS) can characterize the extension of the infiltration. However, CEUS dynamics are not sufficiently specific and may be misinterpreted without appropriate clinical information.

- Early microbiological assessment and treatment of ILAS is mandatory to prevent further metastatic spread.

\section{Conflict of Interest}

The authors declare that they have no conflict of interest.

sis of liver lesions. In a patient with appropriate symptoms, a hypoechoic liver lesion with an edematous rim and without central vascularization is a typical finding of a necrotizing liver abscess formation (R. Barosa et al. J Med Ultrason 2001; 44: 239-245). However, Klebsiella pneumoniae-induced ILAS often appears as an inhomogeneous lesion with irregular demarcation (J. Y. Hui et al. Radiology 2007; 242(3): 769-776), which is caused by the infiltrative growth of this mucoid phenotype. Thus, these lesions could be misclassified as a malignant tumor.

Both diagnosis and therapeutic interventions of liver lesions are often based on contrast-enhanced ultrasound (CEUS). CEUS of classic pyogenic liver abscesses shows no central perfusion, but peripheral enhancement in the arterial phase, frequent- ly followed by a hyper- or isoenhancing rim in the portal venous phase and a hypoenhancing rim in the late phase reflecting central necrosis and surrounding inflammation. This pattern can be accompanied by enhanced septa in some cases (C. F. Dietrich et al. Ultraschall in Med 2020). However, depending on the stage of the phlegmonous inflammation, diffuse hyperenhancement or non-enhancing foci may challenge further differential diagnosis.

Regarding the hvKp scenario, a recent case series has described septal arterial enhancement in three patients but did not analyze the CEUS dynamics in the following phases (G. Whang et al. J Ultrasound Med 2020; 39(7): 1447-1452). In our patient, we observed an inhomogeneous arterial enhancement followed by a rapid washout phenomenon of hvKp-induced ILAS. Histologic evidence showed an irregular spread

\section{Acknowledgement}

The authors acknowledge support from Leipzig University for Open Access Publishing.

\section{Authors}

Tobias Bielow ${ }^{1}$, Valentin Blank ${ }^{1}$, Sabine Opitz ${ }^{2}$, Holger Gößmann³ ${ }^{3}$, Martin Hecker ${ }^{1}$, Daniel Seehofer ${ }^{4}$, Christoph Lübbert ${ }^{5}$, Thomas Karlas ${ }^{1}$ 


\section{Affiliations}

1 Division of Gastroenterology, Department of Medicine II, University Hospital Leipzig, Leipzig, Germany

2 Institute of Pathology, Department of Diagnostics, University Hospital Leipzig, Leipzig, Germany

3 Department of Diagnostic and Interventional Radiology, University Hospital Leipzig, Leipzig, Germany

4 Visceral, Transplant, Thoracic und Vascular Surgery Center, University Hospital Leipzig, Leipzig, Germany

5 Division of Infectious Diseases and Tropical Medicine, Department of Medicine II, University Hospital Leipzig, Leipzig, Germany

\section{Correspondence}

Thomas Karlas

Department of Internal Medicine II, Division of Gastroenterology, Universitatsklinikum Leipzig Liebigstraße 20

Leipzig 04103

Germany

Tel.: + 493419712240

thomas.karlas@medizin.uni-leipzig.de published online 2021

\section{Bibliography}

Ultrasound Int Open 2021; 7: E2-E5

DOI 10.1055/a-1471-6907

ISSN 2199-7152

(C) 2021. The Author(s)

This is an open access article published by Thieme under the terms of the Creative Commons

Attribution-NonDerivative-NonCommercial-License, permitting copying and reproduction so long as the original work is given appropriate credit. Contents may not be used for commecial purposes, or adapted, remixed, transformed or built upon. (https:/ creativecommons. org/licenses/by-nc-nd/4.0/) Georg Thieme Verlag KG, Rüdigerstraße 14, 70469 Stuttgart, Germany

(ㄷ)(1) $\odot \odot$ 